\title{
Cost-Benefit Analysis of Electric Bus Fleet with Various Operation Intervals
}

\section{Vepsäläinen, Jari}

IEEE

2018

Vepsäläinen , J , Baldi , F , Lajunen , A , Kivekäs , K \& Tammi , K 2018 , Cost-Benefit

Analysis of Electric Bus Fleet with Various Operation Intervals . in 2018 21st International

Conference on Intelligent Transportation Systems (ITSC) . IEEE International Conference on

Intelligent Transportation Systems-ITSC , IEEE , pp. 1522-1527, International Conference

on Intelligent Transportation Systems (ITSC), Maui, Hawaii , United States , 04/11/2018 . https://doi.org/10.1109/ITS

http://hdl.handle.net/10138/312789

https://doi.org/10.1109/ITSC.2018.8569583

unspecified

acceptedVersion

Downloaded from Helda, University of Helsinki institutional repository.

This is an electronic reprint of the original article.

This reprint may differ from the original in pagination and typographic detail.

Please cite the original version. 


\section{Cost-Benefit Analysis of Electric Bus Fleet with Various Operation Intervals}

\author{
Jari Vepsäläinen \\ Department of Mechanical Engineering \\ Aalto University \\ Espoo Finland \\ jari.vepsalainen@aalto.fi
}

\author{
Francesco Baldi \\ SCI STI FM Group \\ Ecole Polytechnique Federale de Lausanne \\ Sion, Switzerland \\ francesco.baldi@epfl.ch
}

\author{
Antti Lajunen \\ Department of Mechanical Engineering \\ Aalto University, Finland \\ Deparment of Agricultural Sciences \\ University of Helsinki, Finland \\ antti.lajunen@helsinki.fi
}

\author{
Klaus Kivekäs \\ Department of Mechanical \\ Engineering \\ Aalto University \\ Espoo Finland \\ klaus.kivekas@aalto.fi
}

\author{
Kari Tammi \\ Department of Mechanical \\ Engineering \\ Aalto University \\ Espoo Finland \\ kari.tammi@aalto.fi
}

\begin{abstract}
Electric buses are particularly suitable for city and suburban routes due to zero local exhaust and noise emissions. The operation schedule interval defines the charging power, bus fleet size and total cost of ownership of a bus. We propose a novel cost-benefit method for the scheduling of an electric city bus fleet on a single route. Three different charging infrastructure scenarios were considered. In the first scenario, only one charging station was used. The second scenario considered two charging stations that were located at the same terminus. In the third scenario, two charging stations were located at opposite terminuses. The costs and utilization rates of the buses were analyzed with operation intervals up to 40 minutes. The first scenario with a single charging station had the lowest costs for the entire bus fleet system when the utilization rate was considered. Furthermore, the results show that certain schedule intervals are more cost-beneficial in terms of vehicle specific life-cycle costs than others. In the future, the proposed method is expanded to aid the design of bus network scheduling under energy demand uncertainty.
\end{abstract}

Keywords - Electric Vehicles; Infrastructure for Charging, Communication and Controls; Planning

\section{INTRODUCTION}

\section{A. Motivation}

Electric city buses bestow enormous potential for short distance routes in highly populated areas, since they are efficient, quiet and have zero local emissions. However, high investment costs and range-anxiety due to low specific energy of vehicle batteries are in the way of large-scale electrification of public transportation. These two limitations are contradictory because larger battery can reduce range anxiety but also increases the initial investment costs. The battery capacity of an electric city bus can vary e.g. from 60 to $548 \mathrm{kWh}$ [1] depending on the operating schedule, charging power, battery chemistry and bus size.

Most commonly used buses are 12 meters long and have occupancy for 72-96 persons [2]. Traditional diesel buses weigh around 12000-13000 kg [1], whereas lightweight chassis of $8500 \mathrm{~kg}$ [2] is more viable for an electric city bus. For lightweight electric city buses, end terminus charging has been shown to have lower life-cycle costs (LCC) than opportunity charging or overnight charging strategies on city and suburban routes [3]. End terminus charging refers to recharging at the start or end terminus on every driving cycle. High-power lithium titanate batteries (LTO) are best suited to end terminus charging because they are eligible for high current charging [4], [5]. However, even if the bus size and battery chemistry are predetermined, the operation schedule and charging power vary case by case.

We present a new approach to analyze the costs and benefits of electric bus fleet operation in respect to schedule interval, charging power and battery capacity. Different operation schedule intervals are studied for three charging scenarios.

- In the first scenario, only one charging station is considered, which is located at the terminus A.

- In the second scenario, two charging stations are placed at the same terminus, $\mathrm{A}$.

- In the third scenario, two charging stations are placed at different terminuses (A and B).

The analysis is focused on minimizing the total cost of ownership (TCO) depending on the operation schedule. The bus size, route duration and energy consumption are 
constant to make fair comparisons. Even though we only consider a case example, and the results are not universal, it gives reference of the effect of charging station positioning to the life-cycle costs.

\section{B. State-of-the-art}

Electric bus powertrain designs [6]-[9] and comparisons to diesel-powered buses [3], [4], [10], [11] have been previously investigated to evaluate LCC and energy consumption in different scenarios. Even entire bus fleets have been studied, mainly focusing on the recharging systems and scheduling. Wang et al. [12] forecasted that the return of investment time for electric city bus charging infrastructure is still very long and that battery swapping strategy is even more expensive. Rogge et al. [13] determined that $80 \%$ of the existing bus service trips in Muenster, Germany could be operated with electric buses if the charging power capability was $500 \mathrm{~kW}$. They also pointed out that decrease in passenger capacity relieves space for a bigger battery, resulting in reduced charging power, as it allows a more flexible recharge schedule. The wait time at a terminus can be then fully utilized since full charge is rarely achieved with an oversized battery. Rothgang et al. [5] continued the research by highlighting that the battery and charging systems should be designed for each bus network separately. Sinhuber et al. [9] proposed a modular battery pack to dimension the energy storage of each bus to suit its mission.

In addition to these planning phase studies, Wang et al. [14] proposed a cost-optimal recharging schedule for a bus network. They showed that the total costs of the bus fleet can be reduced by optimal scheduling. However, they used a fixed charging time and only considered one charging station per charging location. Sebastiani et al. [15] conducted a case study of real-world bus network operation, where the focus was on the optimal placement of charging stations for opportunity charging. Opportunity charging refers to a charging strategy where the bus is charged at some of the bus stops while picking up passengers. Increasing the number of charging stations decreased the time of individual recharge events, which gradually led to a solution where the recharging did not affect the operation schedule at all. With only the minimal amount of charging stations, the passengers had to wait extra time at every charging station stop before the journey continued. Paul \& Yamada [16] developed an algorithm that maximizes the utilization of electric buses in a situation where existing diesel bus lines would be replaced with electric buses. A high charging power was required in order to make the replacement worthwhile. Prohaska et al. [17] measured the energy consumption and recharging behavior of an electric city bus during 2014-2015. They noted that the road-grade and heating, ventilation and air conditioning (HVAC) power must be considered while designing the energy storages and recharging schedules of electric city bus fleets.

\section{METHODS}

The bus route 23 operating at Helsinki, Finland, was taken as a case example. The route has small differences depending on the direction, as can be seen in Fig. 1. The differences were neglected and the trip from A to B was considered identical to the trip from B to A. The entire route is $16 \mathrm{~km}$ long and it takes 40 minutes to cover it with an average speed of $20 \mathrm{~km} / \mathrm{h}$. The actual operation interval on the route 23 varies between 13-15 minutes.

The charging system dimensioning was based on the LCC calculation method presented in [4]. The calculations were used for both single and multiple charging station systems scenarios. The departure interval of the buses was used as an input. As the interval time reduced, the charging power had to be increased, as well as the number of buses, because there was less time available for recharging. First, the bus fleet size was determined. The number of buses $N_{c}$, wait time used for charging $t_{w}$ and total trip time $t_{\text {rtot }}$, that includes charging time, are determined by

$$
\begin{gathered}
N_{c}=t_{r} / t_{i n t}, \\
t_{w}=N_{c} \cdot t_{i n t}-t_{r}, \\
t_{r t o t}=t_{r}+t_{w},
\end{gathered}
$$

where $t_{r}$ is the time spent driving the round-trip route and $t_{\text {int }}$ is the bus schedule interval. However, if the wait time $\left(t_{w}\right)$ was less than 5 minutes, an additional bus was added to avoid excessive fast-charging.

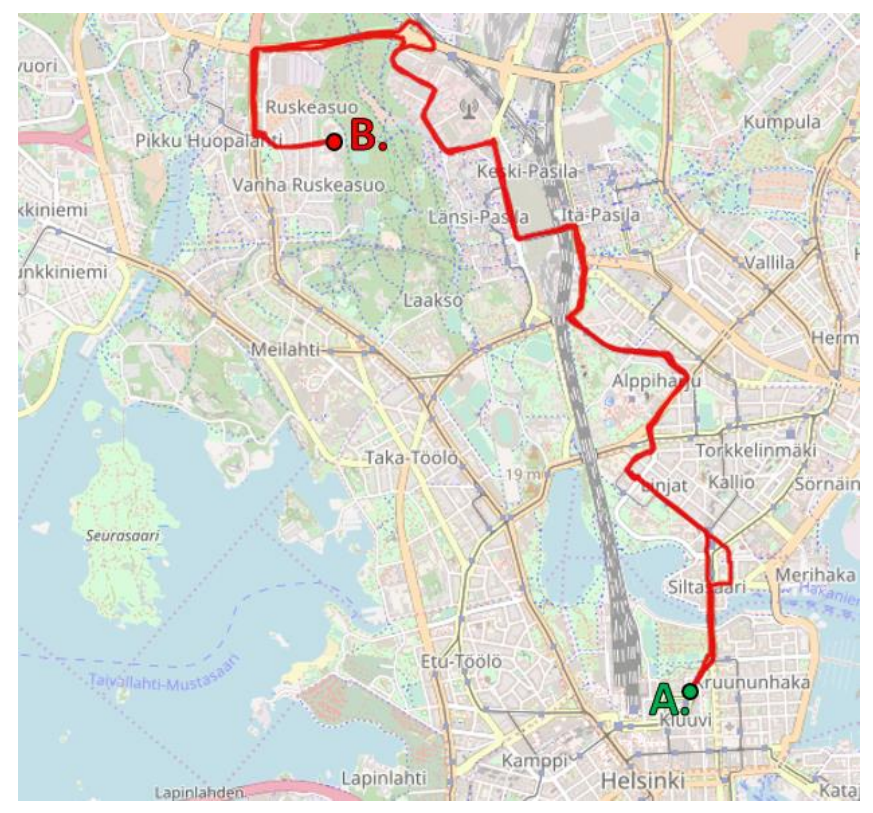

Fig. 1. Helsinki bus route 23 , which is used as a case example. The image was developed with GPSVisualizer [18].

Instead of using a simulation model, an average consumption $E_{\text {avg }}$ and average HVAC system $\left(P_{H V A C, a v g}\right)$ of lightweight electric bus were applied to estimate yearly 
costs of operation. The maximum consumption $E_{\max }$ and power of HVAC system $\left(P_{H V A C, \max }\right)$ were considered when sizing the battery and charging systems. The effect of auxiliary devices and battery weight were considered in the baseline energy consumption. One kilogram of additional weight increases the consumption by approximately 0.1 $\mathrm{Wh} / \mathrm{km}$. The total energy consumed on a predetermined route is

$$
E_{k m, t o t}=\left(E_{a v g}+0.1 \cdot B_{t c} / B_{p e d}\right)+\left(P_{H V A C, a v g} \cdot t_{r} / 3600\right) / d,
$$

where $d$ is the distance travelled (round-trip), $t_{r}$ is the time spent on route, $B_{t c}$ is the battery capacity and $B_{p e d}$ is the battery pack energy density. It was assumed that the HVAC is turned off while recharging the vehicle. The energy consumption calculation parameters are listed in Table I.

TABLE I. Operational parameters of the vehicle.

\begin{tabular}{lll} 
Parameter & Value & Symbol \\
\hline Avg. Energy consumption $(\mathrm{kWh} / \mathrm{km})$ & 0.7 & $E_{\text {avg }}$ \\
Max. Energy consumption $(\mathrm{kWh} / \mathrm{km})$ & 1.4 & $E_{\max }$ \\
Avg. HVAC power $(\mathrm{kW})$ & 5 & $P_{H V A C, \text { avg }}$ \\
Max. HVAC power $(\mathrm{kW})$ & 20 & $P_{H V A C, \max }$ \\
Battery pack energy density $(\mathrm{Wh} / \mathrm{kg})[3]$ & 56 & $B_{\text {ped }}$ \\
Battery cycles & 10000 & $B_{N}$ \\
Operating hours $(\mathrm{h} / \mathrm{y})$ & 4000 & $T_{o p}$ \\
Service life $(\mathrm{y})$ & 12 & $T_{s}$
\end{tabular}

Once the energy demand and wait time are determined, the charging power can be calculated with

$$
P_{\text {chg }}=\left(3600 \cdot E_{\max } \cdot d\right) /\left(t_{w} \cdot \eta_{c h g}\right),
$$

where the charging efficiency $\eta_{\text {chg }}$ is set to 0.85 to account for losses in the recharging event. Also, charging power was limited to a maximum of $400 \mathrm{~kW}$. This is due to the fact that even high-power batteries should not be charged with more than $10 \mathrm{C}$-rate, which was another limitation. C-rate is a measure for battery charge or discharge rate relative to its capacity. In addition, the battery ages with charge-discharge cycles, excessive currents, elevated temperatures and storage time. Only cycle-aging was considered here. Thus, the number of battery replacements during a bus's service life is governed by

$$
N_{b r}=d_{s}\left(B_{N} \cdot B_{t c} / E_{k m, t o t}\right),
$$

where $d_{s}$ is the total distance travelled during service life and $B_{N}$ is the number of cycles the battery can endure before reaching end-of-life (EOL). Once the system is designed, the TCO of an individual bus $C_{b u s}$ can be calculated with

$$
\begin{gathered}
C_{b u s}=\left[N_{c}\left(C_{v}+C_{b}\right)+C_{c}+\Sigma_{j}\left(C_{e} \cdot d_{s} \cdot E_{k m, t o t}+C_{d e m}+C_{b}\left(N_{b r} / T_{s}\right)\right.\right. \\
\left.\cdot\left(1-D_{\text {rate }}\right)^{-j}\right] / N_{c},
\end{gathered}
$$

where $j$ is service years from first to last $\left(T_{s}\right)$ and rest of the economical parameter values are listed in Table II. The investment costs are determined by the vehicle cost $C_{v}$, battery $\operatorname{cost} C_{b}$ and charging system cost $C_{c}$. The yearly operation and replacement costs consist of electricity costs $C_{e}$, demand charges $C_{d e m}$ and battery replacement costs. The demand charges are monthly fees that are based on the maximum power consumed by the charging system. Thus, the demand charges increased significantly if multiple buses were charged simultaneously. All of the yearly costs were discounted with discount rate, $D_{\text {rate }}$.

TABLE II. Economical parameters of the system

\begin{tabular}{lll} 
Parameter & Value & Symbol \\
\hline Vehicle cost [3] $(\mathrm{k} €)$ & 350 & $C_{v}$ \\
Charging station cost [19] $(€ / \mathrm{kW})$ & 350 & $C_{c}$ \\
Battery cost [3], [5] $(€ / \mathrm{kWh})$ & 800 & $C_{b}$ \\
Electricity cost [4] $(€ / \mathrm{kWh})$ & 0.1 & $C_{e}$ \\
Demand cost [4] $((€ / \mathrm{kW}) / \mathrm{y})$ & 120 & $C_{\text {dem }}$ \\
Discount rate $(\mathrm{real})(\%)$ & 5 & $D_{\text {rate }}$
\end{tabular}

\section{RESULTS}

\section{A. First scenario: one charging station}

First, we considered a scenario where only one charging station was installed at the terminus A. The TCO per bus as a function of schedule interval is shown in Fig. 2. Schedule intervals ranged from 1 to 40 minutes, with steps of 1 minute. When the size of the bus fleet was constant, decrease in schedule interval increased the TCO, since charging power demand increases. The markers represent feasible solutions that meet the recharging requirements and the grey line indicates all possible solutions. The schedule intervals under 7 minutes required more than one charging station to be feasible because the charging power demand exceeded the $400 \mathrm{~kW}$ limit. The utilization percentage of the buses in respect to schedule interval is shown in Fig. 3. The utilization rate represents the portion of time that was used for driving the route. The rest of the time was spent on charging. The relation between utilization and operation intervals was approximately linear, as was the charging power demand increase. Also, the lower the utilization rate, the longer the drivers are being paid for sitting idly.

\section{B. Second scenario: two charging stations at the same terminus}

In the second scenario, two charging stations were placed at the terminus A. This doubled the time a single bus can charge compared to the first scenario with only one charging station. However, additional buses were also required because otherwise one of the charging stations would be left unused or at least underutilized. The TCO per bus was lower in this scenario than in the first because the total costs were split between the buses and so additional buses relieved the costs of the individual buses. In Fig. 4, The TCO per bus is clearly lower than in the first scenario at all intervals, yet the utilization of the buses was worse. 


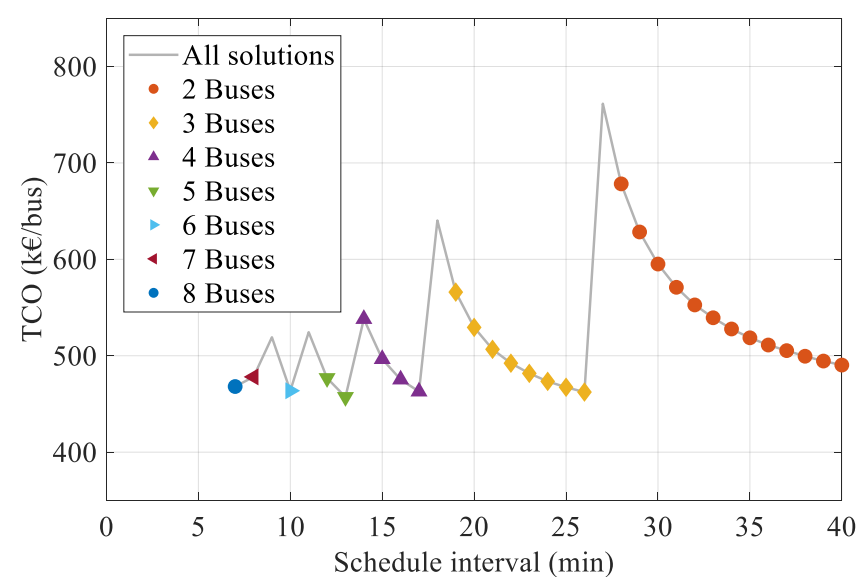

Fig. 2. The TCO of a bus as a function of schedule interval, when only one charging station is used. The peaks and intervals under 7 minutes are not feasible because the fast-charge power $(400 \mathrm{~kW})$ is exceeded.

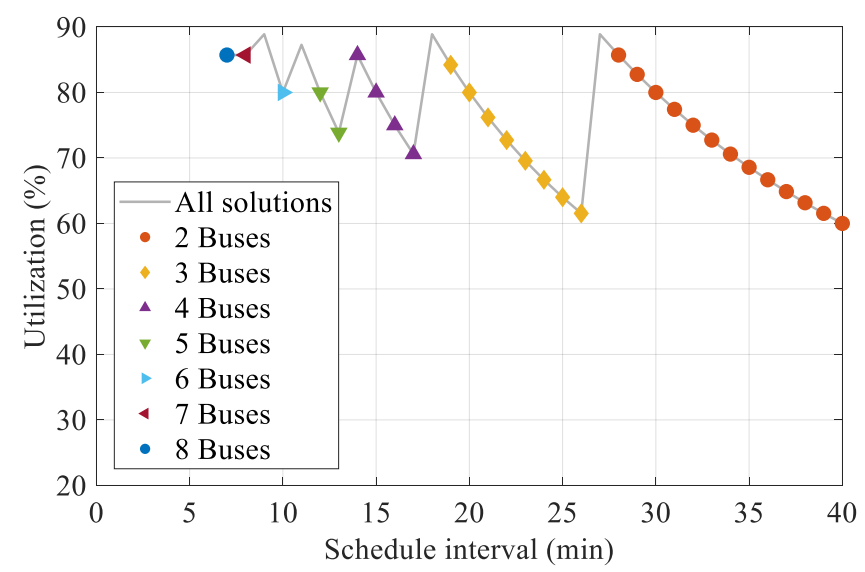

Fig. 3. Utilization rate of a bus as a function of schedule interval, when only one charging station is used.

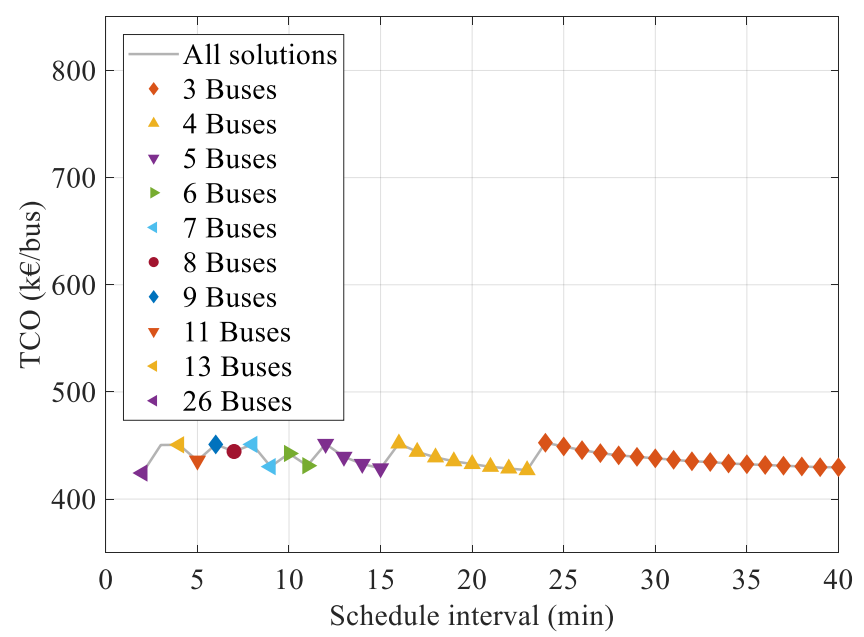

Fig. 4. The TCO of a single bus, in the second scenario where two charging stations are at terminus A.

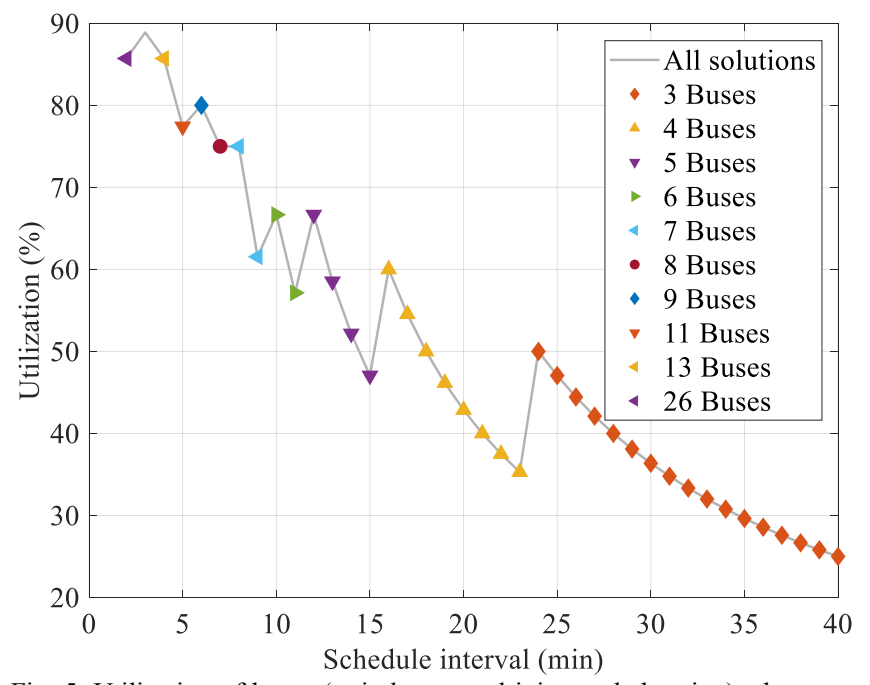

Fig. 5. Utilization of buses (ratio between driving and charging) when two charging stations are at terminus A.

The utilization rate of buses was worse because two charging stations are occupied at all times, compared to having only one, as shown in Fig. 5. The lower utilization rate increases labor costs of drivers significantly but does not have as significant effect if the fleet is operating autonomously.

\section{Third scenario: two charging stations at different terminuses}

The third investigated scenario had two charging stations that were located at different terminuses of the route, $\mathrm{A}$ and B. Terminus B is at the halfway point of the symmetric round-trip, which allows the reduction of the bus energy storage capacity to half of its original value. In Fig. 6, the costs of a bus are notably higher than in the previous scenarios with intervals higher than 27 . This is because the number of buses was at the minimum value of two, yet there were equally as many charging stations. As more buses were introduced with shorter operation intervals, the price of the charging stations was divided more evenly among them. The TCO per bus and utilization rate in this scenario were close to the ones portrayed in the first scenario, as shown in Fig. 6 and Fig. 7. Nevertheless, the utilization rates were systematically lower, especially in the time intervals between 20 and 28 minutes. However, the TCO per bus was lower in this scenario than in the first with operation intervals shorter than 29 minutes. 


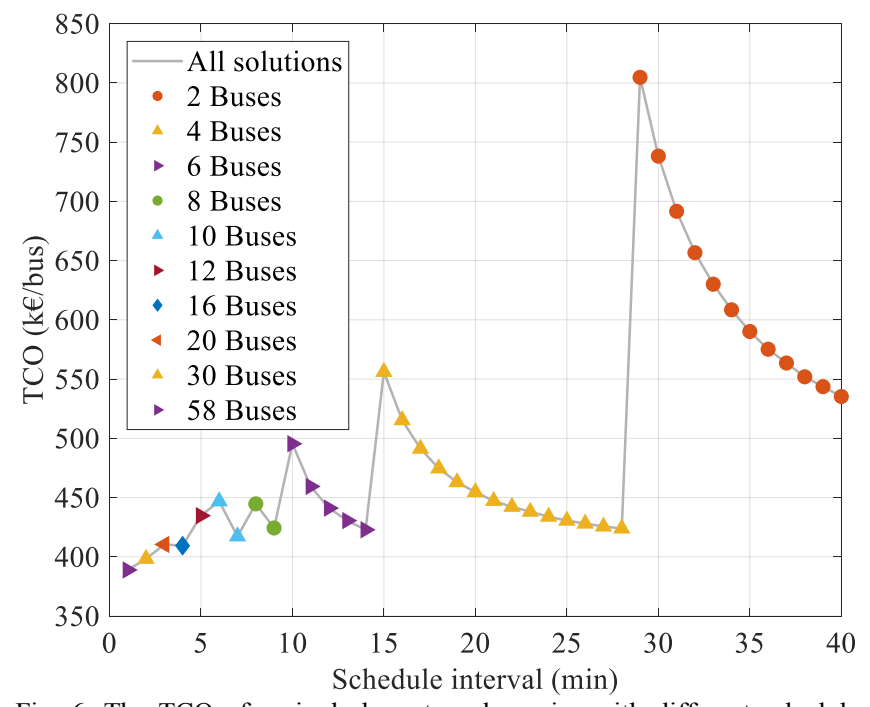

Fig. 6. The TCO of a single bus strongly varies with different schedule intervals when there are charging stations at each of the route terminuses A and $\mathrm{B}$.

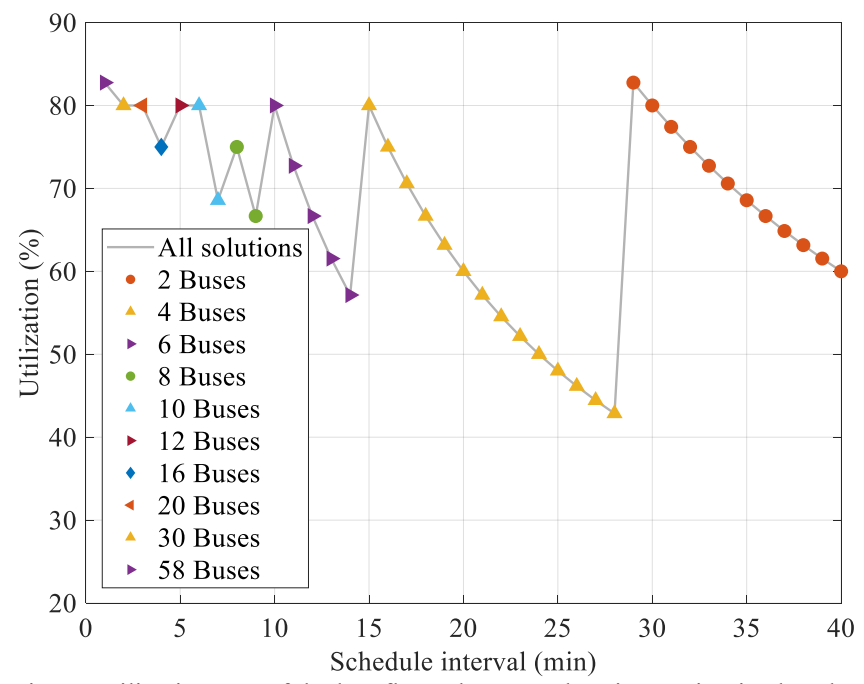

Fig. 7. Utilization rate of the bus fleet when one charging station is placed at both of the terminuses, A and B. The utilization rates with different intervals resemble the rates of the first scenario.

\section{Comparison of scenarios}

In order to compare the three proposed charging scenarios, the total costs of the entire systems in all scenarios are displayed in Fig. 8 with varying operation intervals. Based on the aggressive system cost increase in the short operation intervals, it would be recommendable not to consider schedule intervals less than 5 minutes on this case-study route.

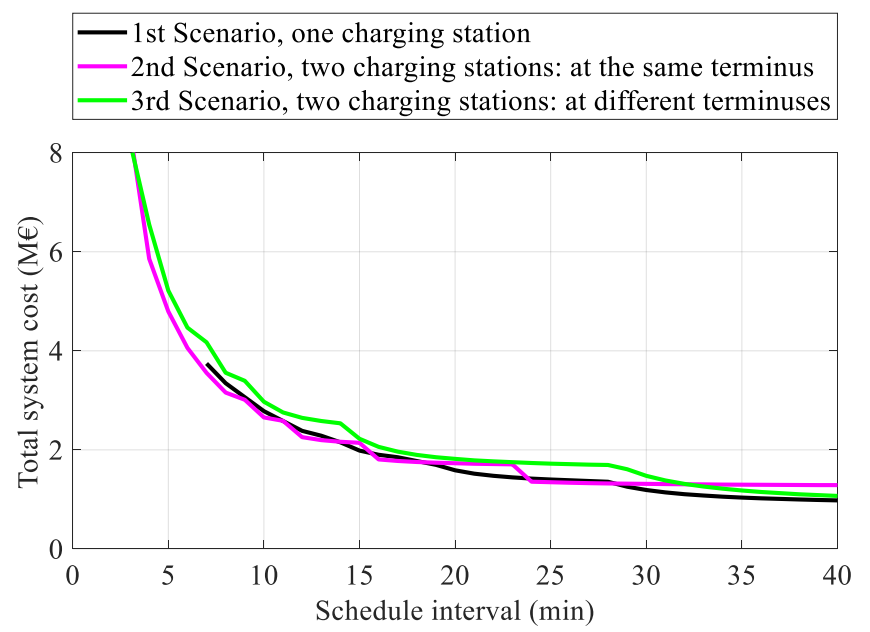

Fig. 8. The total cost of the entire bus fleet system for different scenarios.

The total costs were lowest for the first scenario for most of the operation intervals longer than 15 minutes. The scenarios with two charging stations come close to the same costs at some time intervals, yet the second scenario was the most cost efficient for intervals shorter than 15 minutes. Another advantage of the second scenario was that the Crates were on average lower than in the other scenarios, which made it the most flexible solution. Thus, if two charging stations were installed on the same route, they should be placed at the same terminus. However, the utilization rate was higher for the third scenario than in the second. Taking the utilization rate into account, the best solution was to have only one charging station $\left(1^{\text {st }}\right.$ scenario $)$ and the next best was to have two charging stations placed at separate terminuses ( ${ }^{\text {rd }}$ scenario), at least for nonautonomous operation.

\section{DISCUSSION}

As the results show, the first charging scenario, where only one charging station was purchased, was the most costbeneficial for all operation intervals when the utilization rate was considered. It seems intuitive, but it is interesting that even halving the battery capacity, in the third scenario, did not decrease the costs of the entire operation substantially. Also, certain schedule intervals were more cost-beneficial than others in all scenarios, meaning that with almost the same system price a shorter schedule interval can be achieved. Furthermore, the price per bus was lower in most of the operation intervals in the second and third scenarios compared to the first scenario. However, in these scenarios, the bus fleet was larger as two buses needed to be charged simultaneously instead one. This lead to lower utilization rates but concurrently to reduced charging power. However, multiple charging stations and lower charging power leave leeway for variation in the bus fleets energy consumption and in traffic conditions, which were not taken into account.

In reality, there is variation in the energy demand that is caused by uncertainty of environmental conditions, such as 
ambient temperature and road-grade, in addition to driving style, traffic and auxiliary power usage variations [20]. These uncertainties are unpredictable in the planning phase of operation and charging schedules. With uncertainty quantification, the operation and recharging timetables could adapt to variation in the bus fleet's energy consumption and to varying mission times caused by the ever-changing traffic conditions. We also considered only a single route. More routes need to be studied to verify and generalize the presented LCC calculations. In addition, the costs of logistics, indirect costs and labor costs could be taken into account. In future work, we will focus on adaptable scheduling of an entire bus network, which includes multiple routes with shared charging infrastructure.

\section{CONCLUSION}

Three different charging scenarios were calculated to analyze the cost-benefits of different operation intervals on a single bus route. First scenario had only one charging station and the second two charging stations at the same terminus. In the third scenario, two charging stations were placed at different terminuses. The entire systems cost of ownership was lowest for the first scenario when utilization rate was taken into account. However, the scenarios with two charging stations were potentially more adaptable to variation in the energy demand and traffic conditions. Moreover, some operation schedule intervals were more cost-beneficial than others. The cost of the bus fleet system grew aggressively with operation intervals shorter than 5 minutes and such short intervals should thus be avoided. The operation and scheduling research will be continued by studying a bus network with multiple routes while uncertainty factors are considered.

\section{ACKNOWLEDGMENT}

The research was funded by the Henry Ford Foundation. The authors also acknowledge the financial support from KAUTE and Koivunen Foundations for the research reported in this paper.

\section{REFERENCES}

[1] Z. Gao et al., "Battery capacity and recharging needs for electric buses in city transit service," Energy, vol. 122, pp. 588-600, 2017.

[2] A. Lajunen and K. Tammi, "Energy consumption and carbon dioxide emission analysis for electric city buses," in EVS29 Symposium, 2016, pp. 1-12.

[3] A. Lajunen, "Lifecycle costs and charging requirements of electric buses with different charging methods," J. Clean. Prod., vol. 172, pp. 56-67, 2018.

[4] A. Lajunen, "Energy consumption and cost-benefit analysis of hybrid and electric city buses," Transp. Res. Part C Emerg.
Technol., vol. 38, pp. 1-15, 2014.

[5] S. Rothgang, M. Rogge, J. Becker, and D. U. Sauer, "Battery design for successful electrification in public transport," Energies, vol. 8, no. 7, pp. 6715-6737, 2015.

[6] R. Kühne, "Electric buses - An energy efficient urban transportation means," Energy, vol. 35, no. 12, pp. 4510-4513, 2010.

[7] A. Lajunen, "Powertrain design alternatives for electric city bus," 2012 IEEE Veh. Power Propuls. Conf., pp. 1112-1117, 2012.

[8] M. Mahmoud, R. Garnett, M. Ferguson, and P. Kanaroglou, "Electric buses: A review of alternative powertrains," Renew. Sustain. Energy Rev., vol. 62, pp. 673-684, 2016.

[9] P. Sinhuber, W. Rohlfs, and D. U. Sauer, "Study on power and energy demand for sizing the energy storage systems for electrified local public transport buses," 2012 IEEE Veh. Power Propuls. Conf., no. January 2012, pp. 315-320, 2012.

[10] A. Lajunen and T. Lipman, "Lifecycle cost assessment and carbon dioxide emissions of diesel, natural gas, hybrid electric, fuel cell hybrid and electric transit buses," Energy, vol. 106, pp. 329-342, 2016.

[11] J. Vepsäläinen, "Driving Style Comparison of City Buses: Electric vs. Diesel," in IEEE Vehicle Power and Propulsion Conference, 2017.

[12] N. Wang, Y. Li, and Y. Liu, "Economic evaluation of electric bus charging infrastructure," in 17th International IEEE Conference on Intelligent Transportation Systems (ITSC), 2014, pp. 27992804.

[13] M. Rogge, S. Wollny, and D. U. Sauer, "Fast charging battery buses for the electrification of urban public transport-A feasibility study focusing on charging infrastructure and energy storage requirements," Energies, vol. 8, no. 5, pp. 4587-4606, 2015.

[14] Y. Wang, Y. Huang, J. Xu, and N. Barclay, "Optimal recharging scheduling for urban electric buses: A case study in Davis," Transp. Res. Part E Logist. Transp. Rev., vol. 100, pp. 115-132, 2017.

[15] M. T. Sebastiani, R. Luders, and K. V. O. Fonseca, "Evaluating Electric Bus Operation for a Real-World BRT Public Transportation Using Simulation Optimization," IEEE Trans. Intell. Transp. Syst., vol. 17, no. 10, pp. 2777-2786, 2016.

[16] T. Paul and H. Yamada, "Operation and charging scheduling of electric buses in a city bus route network," in 17th IEEE International Conference on Intelligent Transportation Systems, ITSC 2014, 2014, vol. 1, pp. 2780-2786.

[17] R. Prohaska, K. Kelly, and L. Eudy, "In-Use Fleet Evaluation of Fast-Charge Battery Electric Transit Buses," in IEEE Transportation Electrification Conference, 2016.

[18] “GPSVisualizer," Accessed on 11th of April 2018. [Online]. Available: http://www.gpsvisualizer.com.

[19] I. Mareev, J. Becker, and D. U. Sauer, "Battery dimensioning and life cycle costs analysis for a heavy-duty truck considering the requirements of long-haul transportation," Energies, vol. 11,55, no. $1,2018$.

[20] J. Vepsäläinen, K. Kivekäs, K. Otto, and K. Tammi, "Development and validation of energy demand uncertainty model for electric city buses," Transp. Res. Part D Transp. Environ., 2018. 